\title{
Radiation Processes in the Universe: Synchrotron Radiation and Propagation Effects
}

\author{
Denise Gabuzda* \\ Department of Physics, University College Cork, Ireland \\ E-mail: gabuzda@phys.ucc.ie
}

This chapter provides a basic introduction to relativistic effects manifest in observations of energetic directed flows, such as the relativistic jets of Active Galactic Nuclei or microquasars; synchrotron radiation, which is among the most commonly observed and distinctive types of non-thermal radiation encountered observationally; and the propagation effects Faraday rotation, depolarization and conversion, which can affect polarized radiation that passes through a region with magnetized plasma. This is not meant to be a full, theoretically rigorous treatment, but instead to provide some qualitative feeling for the nature of the various effects and processes considered.

2nd MCCT-SKADS Training School. Radio Astronomy: fundamentals and the new instruments August 26- September 4, 2008

Sigüenza, Spain

${ }^{*}$ Speaker. 


\section{Relativistic Effects in Observations of Directed Flows}

\subsection{Background}

In differential form, the Lorentz transformations relating the coordinates of an object in one frame (unprimed) and its coordinates in another frame (primed) that moves relative to the first frame with velocity $v$ along the $x$ axis are

$$
\begin{aligned}
d x & =\frac{d x^{\prime}+v d t^{\prime}}{\sqrt{1-v^{2} / c^{2}}} \\
d y & =d y^{\prime} \\
d z & =d z^{\prime} \\
d t & =\frac{d t^{\prime}+v\left(d x^{\prime} / c^{2}\right)}{\sqrt{1-v^{2} / c^{2}}}
\end{aligned}
$$

These can be used directly to find the $x, y$ and $z$ velocity components in the unprimed frame in terms of the corresponding components in the primed frame:

$$
\begin{aligned}
u_{x} \equiv \frac{d x}{d t} & =\frac{u_{x}^{\prime}+v}{1+u_{x}^{\prime} v / c^{2}} \\
u_{y} \equiv \frac{d y}{d t} & =\frac{u_{y}^{\prime}}{\left(1+u_{x}^{\prime} v / c^{2}\right) \gamma} \\
u_{z} \equiv \frac{d z}{d t} & =\frac{u_{z}^{\prime}}{\left(1+u_{x}^{\prime} v / c^{2}\right) \gamma} \\
\gamma & \equiv\left(\sqrt{1-v^{2} / c^{2}}\right)^{-1}
\end{aligned}
$$

The $y$ and $z$ components transform in precisely the same way, since the relative velocity $v$ is purely along the $x$ axis. This means that we can without losing any generality insteady simply consider the components of the motion parallel to and perpendicular to the relative velocity $\mathbf{v}$ between the frames.

Suppose a particle moves with velocity $\mathbf{u}^{\prime}$ in a frame that moves with velocity $\mathbf{v}$ relative to our (unprimed) frame. The observed components of the particle's velocity in our frame parallel and perpendicular to $\mathbf{v}$ are then

$$
\begin{gathered}
u_{\|}=\frac{u_{\|}^{\prime}+v}{\left(1+v u_{\|}^{\prime} / c^{2}\right)} \\
u_{\perp}=\frac{u_{\perp}^{\prime}}{\gamma\left(1+v u_{\|}^{\prime} / c^{2}\right)}
\end{gathered}
$$

The angles along which the particle is observed to move in the two frames are therefore related by the expression:

$$
\begin{aligned}
\tan \theta & =\frac{u_{\perp}}{u_{\|}} \\
& =\frac{u_{\perp}^{\prime}}{\gamma\left(u_{\|}^{\prime}+v\right)}
\end{aligned}
$$



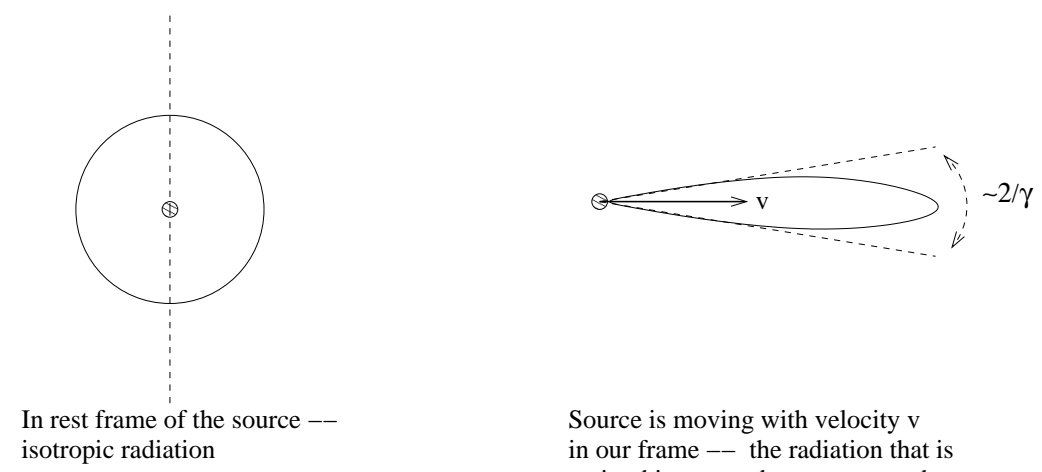

Source is moving with velocity $\mathrm{v}$

in our frame -- the radiation that is

emitted is seen to be concentrated

(beamed) in the direction of the motion

Figure 1: Schematic of Doppler beaming, or boosting, of radiation emitted by a relativistically moving source.

$$
=\frac{u^{\prime} \sin \theta^{\prime}}{\gamma\left(u^{\prime} \cos \theta^{\prime}+v\right)}
$$

\subsection{Aberration and Relativistic Beaming}

For a photon, for which $u^{\prime}=c$, the above relations reduce to

$$
\begin{aligned}
\tan \theta & =\frac{\sin \theta^{\prime}}{\gamma\left(\cos \theta^{\prime}+\beta\right)} \\
\cos \theta & =\frac{\cos \theta^{\prime}+\beta}{1+\beta \cos \theta^{\prime}}
\end{aligned}
$$

Thus, a photon emitted at right angles to $\mathbf{v}$ in its rest frame $\left(\theta^{\prime}=\pi / 2\right)$ is observed at the angle

$$
\begin{aligned}
\tan \theta & =\frac{1}{\gamma \beta} \\
\cos \theta & =\beta \\
\sin \theta & =\frac{1}{\gamma}
\end{aligned}
$$

We say that the source radiation is beamed in the forward direction. When $\gamma$ is large, $\sin \theta$ will be small, and

$$
\theta \simeq \frac{1}{\gamma}
$$

Thus, half the radiation emitted by an isotropically radiating, relativistically moving source is observed within a narrow cone of half-angle $1 / \gamma$. The radiation patterns for a non-moving and moving source will schematically look as is shown in Fig. 1.

This Doppler beaming or Doppler boosting is believed to explain the one-sided appearance of relativistic jets in quasars. In reality there are two jets - one approaching (Doppler boosted), one receding (Doppler dimmed) - if the boosting factor is high enough, the receding jet (whose radiation is beamed in the direction away from the Earth) may be undetectable.

There is another important effect that can be manifest in observations of an object moving relativistically at a small angle to the line of sight. When the viewing angle $\theta<1 / \gamma$, then $\theta^{\prime}<90^{\circ}$, 


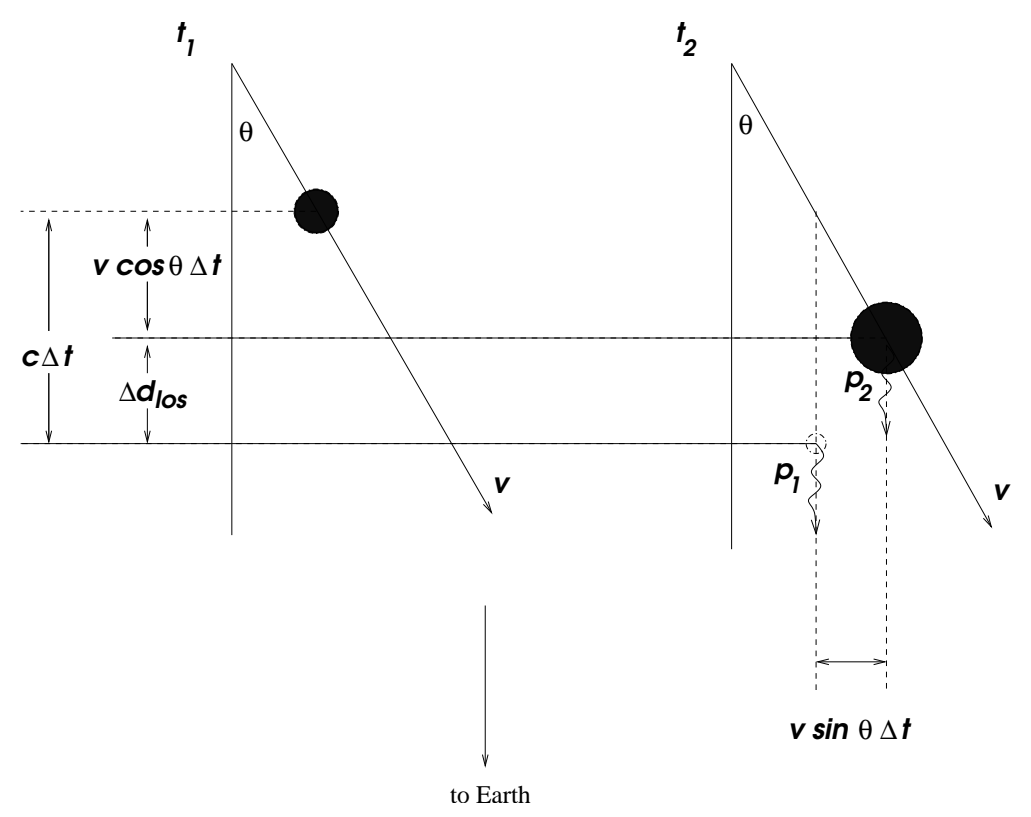

Figure 2: Geometry for superluminal motion.

and we have a "head-on" view of the object; whereas $\theta^{\prime}>90^{\circ}$ when $\theta>1 / \gamma$, and we have a "tail-on" view of the object. In other words, in the latter case, we are detecting photons emitted by the source roughly away from the direction toward the Earth. Since some of the relativistic jets observed in Active Galactic Nuclei (AGN), for example, are viewed at angles $\theta>1 / \gamma$, this certainly affects observations of these objects - although unfortunately, we cannot be sure which ones they are.

\subsection{Superluminal motions}

Consider the motion of a clump of plasma in a jet roughly toward the Earth at two times $t_{1}$ and $t_{2}$. The angle of the clump's motion relative to the direction toward the Earth is $\theta$, and the clump's velocity is $v$ (Fig. 2).

The distance travelled by the clump toward the Earth between the two times is $v \cos \theta \Delta t$, where $\Delta t=t_{2}-t_{1}$. The distance travelled by a photon emitted at $t_{1}$ in the time $\Delta t$ is just $c \Delta t$. The distance between the photons $p_{1}$ and $p_{2}$ will be $c \Delta t-v \cos \theta \Delta t$, and so the measured time between the arrival of photons $p_{1}$ and $p_{2}$ emitted at time $t_{1}$ and $t_{2}$ will be $\Delta t_{\text {meas }}=(1-\beta \cos \theta) \Delta t$, where $\beta=v / c$. The observed distance travelled by the clump in the plane of the sky will be $d_{s k y}=v \sin \theta \Delta t$. Thus, the apparent speed of the clump in the plane of the sky will be

$$
\begin{aligned}
& \beta_{\text {app }}=\frac{\Delta d_{\text {sky }}}{c \Delta t_{\text {meas }}} \\
& \beta_{\text {app }}=\frac{\beta \sin \theta}{1-\beta \cos \theta}
\end{aligned}
$$

We can find the maximum of this function by taking its derivative and setting it equal to zero. This yields a maximum when

$$
\cos \theta=\beta
$$




$$
\sin \theta=\sqrt{1-\cos ^{2} \theta}=\frac{1}{\gamma}
$$

The corresponding maximum apparent speed in the plane of the sky is

$$
\beta_{\max }=\beta \gamma
$$

Clearly, the observed motion will be superluminal, or apparently faster than light, for sufficiently high intrinsic speeds $\beta$ and sufficiently small viewing angles $\theta \sim 1 / \gamma$. This provides a simple explanation of superluminal motions in AGN jets, which are very common; unfortunately, it is not possible to unambiguously disentangle the contributions of $\beta$ and $\theta$ to the observed speeds. Nevertheless, studies of superluminal jet component speeds for samples of AGN are extremely useful in determining the nature of differences between different types of AGN, for example.

\subsection{Measured Variation Timescales and the Doppler Factor}

We saw above that the measured time between the arrival of two photons emitted by a source moving toward the observer at two times separated by $\Delta t$ is

$$
\Delta t_{\text {meas }}=(1-\beta \cos \theta) \Delta t
$$

Here, $\Delta t$ is the time interval in the frame of the observer, and will differ from the time interval between the emission of the two photons in the rest frame of the source (which we denote with a prime) due to relativistic time dilation:

$$
\Delta t=\gamma \Delta t^{\prime}
$$

Therefore,

$$
\Delta t_{\text {meas }}=(1-\beta \cos \theta) \gamma \Delta t \equiv \frac{1}{D} t^{\prime}
$$

The inferred time interval between the arrival of the two photons will be too small by a factor

$$
D=\frac{1}{\gamma(1-\beta \cos \theta)}
$$

The quantity $D$ is called the Doppler factor, and relates quantities in the rest frame of the source and the observer's frame, taking into account both special relativistic effects and the direction of the source's motion relative to the observer. It is essentially a generalized form of the Lorentz factor $\gamma$, and when $\theta=90^{\circ}, D$ reduces to $1 / \gamma$.

For example, if $\theta$ is small enough that $\cos \theta \simeq 1$ and in addition $\beta \simeq 1$ (highly relativistic motion nearly directly toward the Earth),

$$
\begin{aligned}
\Delta t_{\text {meas }} & =\frac{(1-\beta)}{\sqrt{1-\beta^{2}}} \Delta t^{\prime} \\
& =\frac{\sqrt{1-\beta}}{\sqrt{1+\beta}} \Delta t^{\prime} \\
& =\frac{1}{(1+\beta) \gamma} \Delta t^{\prime} \\
\Delta t_{\text {meas }} & \simeq \frac{1}{2 \gamma} \Delta t^{\prime}
\end{aligned}
$$


We will infer a time between the emission of the two photons that is much too short, by a factor of $1 / 2 \gamma$.

Note how important it is to take into account the motion of the source - if time dilation acts alone $\left(\cos \theta=90^{\circ}\right)$, the time interval measured in the observer's frame should be too long by a factor $\gamma$, while if the source is moving roughly toward the observer, the measured time interval will be too short by a similar factor.

\section{Non-Thermal Continuum Radiation: Synchrotron Radiation in a Nutshell}

Whenever any charged particle is accelerated, it will emit radiation. Consider the instantaneous rest frame of the electron, which we will denote with a prime. The electron will not remain at rest in this frame, because it is accelerating, but moves non-relativistically for infinitesimally neighbouring times. This means that Larmor's formula can be used to find the radiated power $P$ in the instantaneous rest frame of the electron:

$$
P=\frac{2 e^{2}\left(a^{\prime}\right)^{2}}{3 c^{3}}
$$

where $e$ is the electron charge, $a^{\prime}$ the acceleration felt and $c$ the speed of light.

In fact, the power radiated by the accelerated electron is independent of the frame in which it is measured. Suppose an energy $d W^{\prime}$ is emitted in a time $d t^{\prime}$ in the instantaneous rest frame of the electron. The corresponding energy and time in the unprimed frame, which moves at a velocity $-v$ relative to the electron are

$$
\begin{array}{r}
d W=\gamma d W^{\prime} \quad d t=\gamma d t^{\prime} \\
P=\frac{d W}{d t}=\frac{d W^{\prime}}{d t^{\prime}}=P^{\prime}
\end{array}
$$

Thus, although Larmor's formula is valid only for non-relativistic motion, we can use this formula to find the power radiated by an accelerated charge, even if the charge is moving relativistically in our frame. This is the case for one of the most common types of non-thermal radio emission: synchrotron radiation - radiation by relativistic electrons spiraling in a magnetic field.

The equation of motion of a relativistic electron in a magnetic field will be

$$
\frac{d}{d t}(\gamma m \vec{v})=e \frac{\vec{v}}{c} \times \vec{B}
$$

If we have only a magnetic field, no work is done, and the electron's speed (and therefore $\gamma$ ) will be constant, together with the "pitch angle" $\phi$ between $\vec{v}$ and $\vec{B}$ (Fig. 3). Thus, we have

$$
\begin{aligned}
\vec{a} & =\frac{e}{\gamma m c} \vec{v} \times \vec{B} \\
a & =\frac{e \beta \sin \phi B}{\gamma m}
\end{aligned}
$$

It can be shown using the velocity transformation that the components of the acceleration parallel to and orthogonal to the direction of motion transform as:

$$
\begin{aligned}
a_{\|}^{\prime} & =\gamma^{3} a_{\|} \\
a_{\perp}^{\prime} & =\gamma^{2} a_{\perp}
\end{aligned}
$$




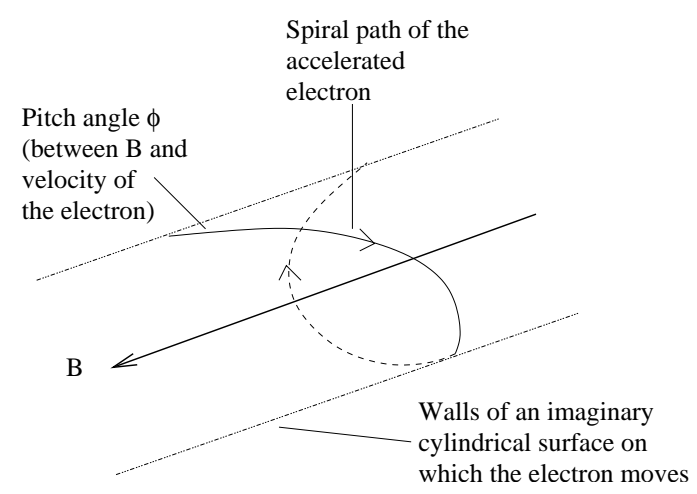

Figure 3: Schematic of synchrotron radiation.

Since we have acceleration only orthogonal to the direction of the motion, the observed synchrotron power in the unprimed frame will be

$$
\begin{aligned}
P & =\frac{2 e^{2}\left(a^{\prime}\right)^{2}}{3 c^{3}} \\
& =\frac{2 e^{4} \gamma^{2} \beta^{2} \sin ^{2} \phi B^{2}}{3 c^{3} m^{2}}
\end{aligned}
$$

If the distribution of pitch angles is uniform (random), this will be after averaging over the pitch angles,

$$
P=\frac{4 e^{4} \gamma^{2} \beta^{2} B^{2}}{9 c^{3} m^{2}}
$$

This is a crude treatment, but yields the general behavior of a synchrotron radiating electrons. The radiation of each electron is highly beamed in the forward direction of its motion, but the total radiation due to all the randomly moving electrons is continuous (not pulsed). Because the power radiated depends inversely on $\mathrm{m}^{2}$, less massive particles will radiate more powerfully; this is why we believe we are dealing primarily with synchrotron radiation by electrons (rather than protons).

We can get an idea of the lifetime of a synchrotron radiating electron by taking the ratio of its energy to the power radiated:

$$
\begin{aligned}
\tau_{\text {synch }} & \simeq E / P \\
& \simeq\left[\gamma m c^{2}\right] /\left[\frac{4 e^{4} \gamma^{2} \beta^{2} B^{2}}{9 c^{3} m^{2}}\right] \\
& \simeq \frac{9 m^{3} c^{5}}{4 e^{4} \beta^{2} \gamma B^{2}}
\end{aligned}
$$

This tells us that more energetic electrons radiate more powerfully and accordingly have shorter lifetimes.

It turns out that most of the power is radiated near the peak frequency

$$
\begin{aligned}
v_{\text {peak }} & \simeq \frac{\gamma_{e B}}{2 \pi m c \beta \sin \phi} \\
& \simeq 3 \times 10^{6} B \gamma^{2} \sin \phi[\mathrm{Hz}]
\end{aligned}
$$


This tells us that more energetic electrons (those with higher $\gamma$ 's) radiate at higher frequencies.

The combination of these effects means that the electrons radiating at higher frequencies have shorter synchrotron lifetimes. This will cause the spectrum radiated by an ensemble of electrons to fall off toward higher frequencies more rapidly (to "steepen") with time, since the electrons giving rise to the higher-frequency radiation lose their energy more rapidly. Regions in which "fresh" energetic electrons have been injected will have relatively less steep (more flat) spectra; this is one way to identify possible regions of "re-acceleration".

Of course, we are dealing with radiation by a population of electrons with some energy distribution. Observationally, the spectra of relatively homogeneous astrophysical objects that emit synchrotron radiation (e.g. supernova remnants like the Crab nebula) are often close to power-laws over substantial ranges of frequency; in other words, the spectrum has the form $S_{v} \propto v^{\alpha}$, where $S_{v}$ is the radiation emitted at frequency $v$. A complete analysis shows that this would come about if the distribution of electron energies is also a power-law:

$$
N(E) d E=N_{o} E^{-p} d E
$$

where $N(E) d E$ is the number density of electrons with energies between $E$ and $E+d E$.

Roughly speaking, if the mean-free-path of a photon in the radiating region is larger than the size of the region, the photon is likely to be able to pass through the region without being absorbed, and we say the region is optically thin. If the mean-free-path of a photon is appreciably less than the size of the region, the photon is likely to be absorbed before it leaves the source region, and we say the region is optically thick. It turns out that, after integrating over the ensemble of electrons, the power radiated at frequency $v$ in the optically thin part of the spectrum is proportional to $v^{\alpha}$, where $\alpha=-(p-1) / 2 . \alpha$ is called the spectral index.

At low frequencies, there can be appreciable absorption of the synchrotron radiation by the radiating electrons themselves; this is called synchrotron self absorption. This makes the radiating region optically thick at these frequencies; it turns out that this gives rise to a slope of $+5 / 2$ in this part of the spectrum. A sketch of the total spectrum for a single homogeneous synchtron-radiating region is shown in Fig. 4 . The theoretical optically thick spectral index is $+5 / 2$; the optically thin spectral index is $\alpha=-(p-1) / 2$. The theoretical optically thick spectral index of $+5 / 2$ is observed very rarely; observed regions are usually only partially optically thick (opaque).

Synchrotron radiation intrinsically has an appreciable degree of linear polarization and low intrinsic circular polarization. If the magnetic field were completely uniform, the electrons were moving in circles in planes perpendicular to the magnetic field (so that the pitch angles are all $90^{\circ}$ ), and we were considering a frequency where the radiation was optically thin, the linear polarization would be $100 \%$. In a more realistic situation, if the magnetic field is completely uniform (ordered), but the distribution of pitch angles is uniform and random, the degree of linear polarization in the optically thin regime can reach about $75 \%$.

More generally, we can think of the radiation as having two components to its linear polarization: one with the plane of the polarization (the polarization $\mathbf{E}$ vector) perpendicular to the magnetic field, and the other with the polarization $\mathbf{E}$ vector parallel to the magnetic field. The former of these two dominates when the radiation is optically thin - qualitatively, this corresponds to $\mathbf{E}$ being in the plane of gyration of the electrons. Recall that the radiation will be optically thick when there is a high probability of a synchrotron photon being absorbed before it can leave the 


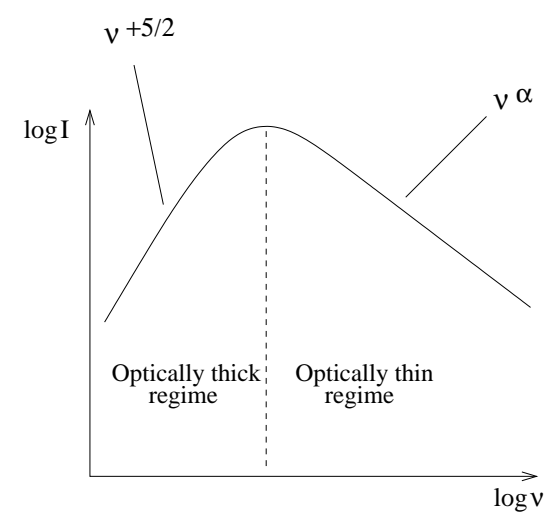

Figure 4: Schematic of the spectrum of a homogeneous source of synchrotron radiation.

region in which it was radiated. In this regime, the component of the linear polarization that has the higher probability of being absorbed is precisely the one that had the higher probability of being emitted in the optically thin regime - the one with $\mathbf{E}$ for the polarization being perpendicular to the $\mathbf{B}$ field in the region where the synchrotron radiation was generated. The other component of the polarization - with its $\mathbf{E}$ vector parallel to the $\mathbf{B}$ field - is less likely to be absorbed, and so more likely to be able to leave the emission region. This means that we have for the two regimes:

- optically thin regime - observed plane of polarization is perpendicular to the $\mathbf{B}$ field and the degree of linear polarization can reach $\simeq 75 \%$

- optically thick regime - observed plane of polarization is parallel to the $\mathbf{B}$ field and the degree of linear polarization is no more than about $10-15 \%$

The reason for the lower degree of polarization for the optically thick regime is that this component of the polarization ( $\mathbf{E}$ parallel to $\mathbf{B}$ ) is less likely to be emitted, so the total fraction of synchrotron photons emitting this component is modest.

\section{Propagation (Faraday) Effects}

\subsection{Faraday Rotation}

Faraday rotation is a rotation of the plane of polarization of an EM wave that occurs if it passes through a region with free electrons and magnetic field. Any EM wave can be described as the sum of any two orthogonal components. In radio astronomy, we usually consider these two components to be right circularly polarized (RCP) and left circularly polarized (LCP). If we have an electron moving at a velocity $\vec{v}$ in an ambient magnetic field $\vec{B}_{o}$, it will feel the Lorentz force

$$
\frac{q \vec{v} \times \vec{B}_{o}}{c}
$$

. This Lorentz force will give rise to gyration of the electron.

Let us suppose that the motion of the electron has been induced by an electromagnetic wave passing through this region, propagating parallel to the field $\mathbf{B}_{o}$. In other words, the velocity of the 
Right- and left-circularly polarized components of EM wave

$\mathrm{RCP}$

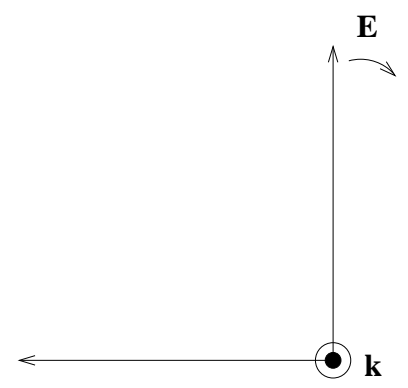

B
LCP

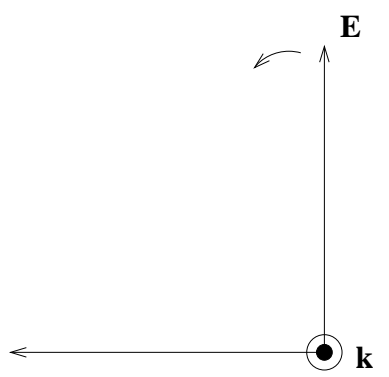

B

ambient $\mathbf{B}_{\mathrm{O}}$

$q \mathbf{v} \times \mathbf{B}_{0}$ (for an electron)

$\mathrm{q} v \mathrm{x} \mathrm{B}$ force is aligned with the rotation of the

EM wave in one case andopposedto it in the other.

Figure 5: Schematic of the interaction between ambient free electrons, the RCP and LCP components of as passing electromagnetic wave and the ambient magnetic field.

electron is initially in the direction of the $\mathbf{E}$ field of this electromagnetic wave. We can describe this electromagnetic wave as being comprised of an RCP and an LCP component, as illustrated in Fig. 5.

In the case of the RCP component, the Lorentz force on the electron is oriented opposite to the direction of rotation of $\vec{E}$, while in the case of the LCP component, the Lorentz force is oriented in the same direction as the rotation of $\vec{E}$.

Thus, we can write the force balance equation

$$
-e \vec{E} \pm \frac{e B_{o} \omega \vec{r}}{c}=-m \omega^{2} \vec{r}
$$

The \pm sign in front of the second term corresponds to the direction of the Lorentz force for the RCP and LCP components of the passing electromagnetic wave, taking into account the fact that the electron charge is negative. The term on the right-hand side is the centripetal force associated with the gyration of the electron.

Solving this equation for $\vec{r}$ gives

$$
\vec{r}=\frac{e}{m}\left(\frac{1}{\omega^{2} \pm \frac{e B_{o} \omega}{m c}}\right) \vec{E}
$$

If the density of electrons in the region is $n$, this will give rise to indices of refraction for the two EM wave components of

$$
\eta=\sqrt{1-\frac{4 \pi n e^{2}}{m \omega\left(\omega \pm \frac{e B_{o}}{m c}\right)}}
$$


Vacuum: equal velocities for RCP \& LCP<smiles>C1CCCCCCCCCCCC1</smiles>

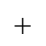

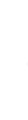

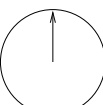

7
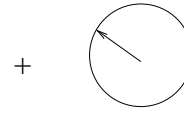

$\longrightarrow$

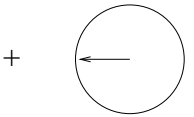

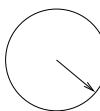

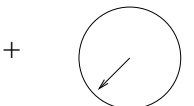

1

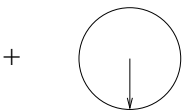

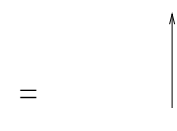

$=$

$=$

\section{$=$}

$=$

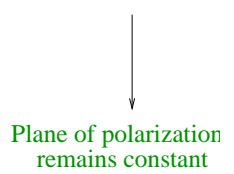

Different velocities for RCP \& LCP
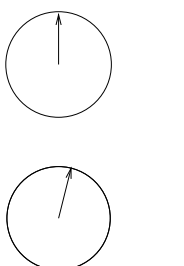

$+$<smiles>CC12CCCC(CC1)CC2</smiles>

$=$

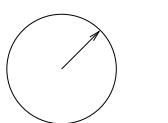

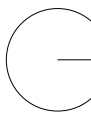

$+$

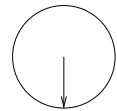

$=$
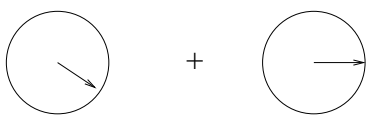

$=$

Figure 6: Schematic illustration of how a delay between the RCP and LCP components of an electromagnetic wave gives rise to a rotation of the plane of linear polarization.

Since the indices of refraction for the RCP and LCP waves will be different, the speeds with which they propagate through the medium will also be different.

- When the wave propagates through a vacuum, the $\mathbf{E}$ vectors for these two components rotate in opposite directions at the same rate, preserving the orientation of the plane of linear polarization, $\chi$ (Fig. 6 , left).

- When the wave propagates through a region with free electrons and an ambient magnetic field with a component in the direction of propagation, the speeds of the RCP and LCP waves become different.

- This causes the plane of polarization to rotate (Fig. 6, right): this is Faraday rotation.

- It turns out that the amount of rotation depends on the strength of the ambient magnetic field, the number density of electrons in the plasma, the electron charge and mass, and the wavelength of the radiation as:

$$
\begin{aligned}
& \Delta \chi=\frac{e^{3} \lambda^{2}}{2 \pi m^{2} c^{4}} \int n(s) \overrightarrow{B_{o}} \overrightarrow{(s)} \cdot d s \\
& \Delta \chi \propto \lambda^{2}
\end{aligned}
$$

- Thus, the action of Faraday rotation can be identified through the $\lambda^{2}$ dependence of the angle $\chi$. 

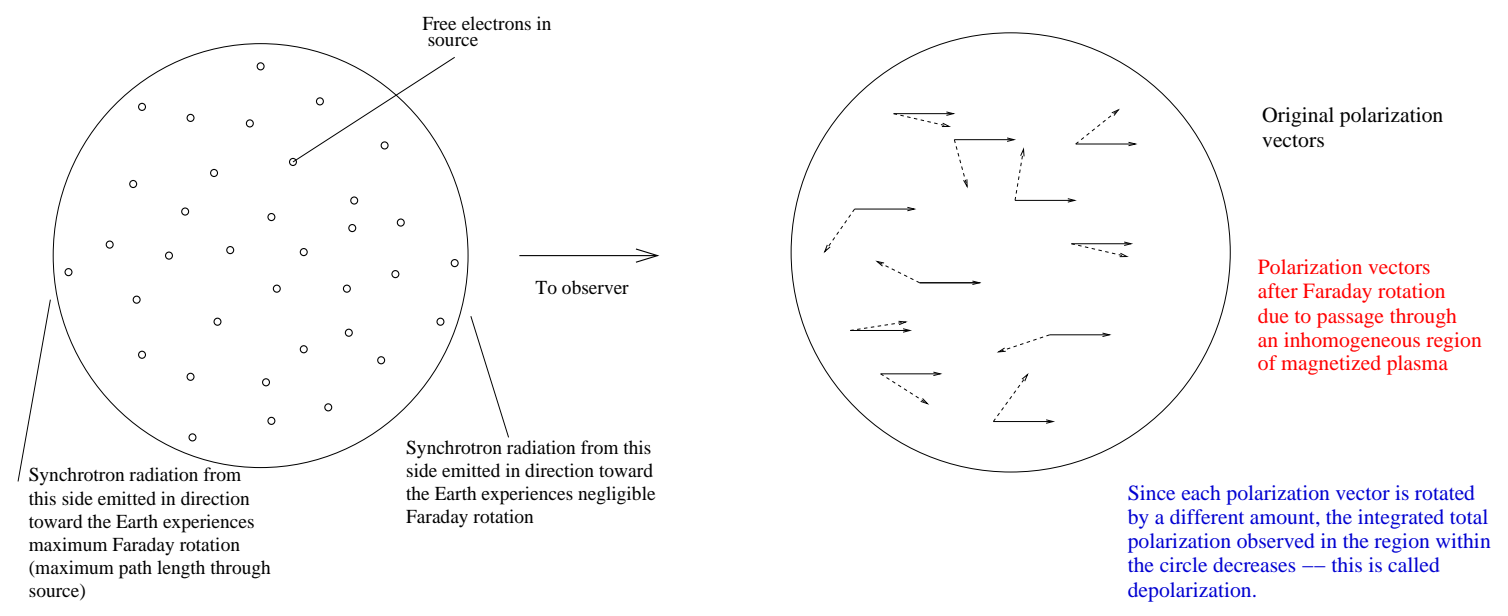

Figure 7: Schematics of front-back depolarization (left) and beam depolarization (right).

Note that it is the line-of-sight component of the ambient magnetic field that determines the magnitude and sign of the Faraday rotation. Essentially all observations of extragalactic sources are affected by Faraday rotation to some extent, because their radiation must always pass through our own Galaxy on its way to the Earth. Usually, these rotations are not very large at wavelengths of about $6 \mathrm{~cm}$ and shorter.

\subsection{Depolarization}

Faraday rotation can also give rise to depolarization of the radiation in association with two effects.

If the source is optically thin (little absorption of the radiation within the source), the radiation emitted at different depths in the source will pass through different amounts of the source volume on its way toward the observer. Therefore, if there are free electrons in the source volume, radiation emitted at different depths in the source will experience different amounts of Faraday rotation on its way through the source volume toward the observer (Fig. 7, left).

Observations have some finite resolution, and so represent the sum of many EM waves propagating along many different lines of sight through the plasma. If appreciable inhomogeneities are present in the plasma electron density and/or ambient magnetic field on scales smaller than the resolution of the observations, different lines of sight will experience different rotation measures (Fig. 7 right).

The depolarization occurs because the total polarization is the sum of the polarizations for multiple regions or lines of sight that experience different Faraday rotations. Because the rotation of the polarization vectors is greater at longer wavelengths, the depolarization will also increase at longer wavelengths. Depolarization is indicated by a decrease in the observed degree of polarization with increasing wavelength.

\subsection{Faraday Conversion}

Incoherent synchrotron radiation produces a very small amount of circular polarization at frequencies of several to tens of gigahertz, and the observed degrees of circular polarization in AGN, 

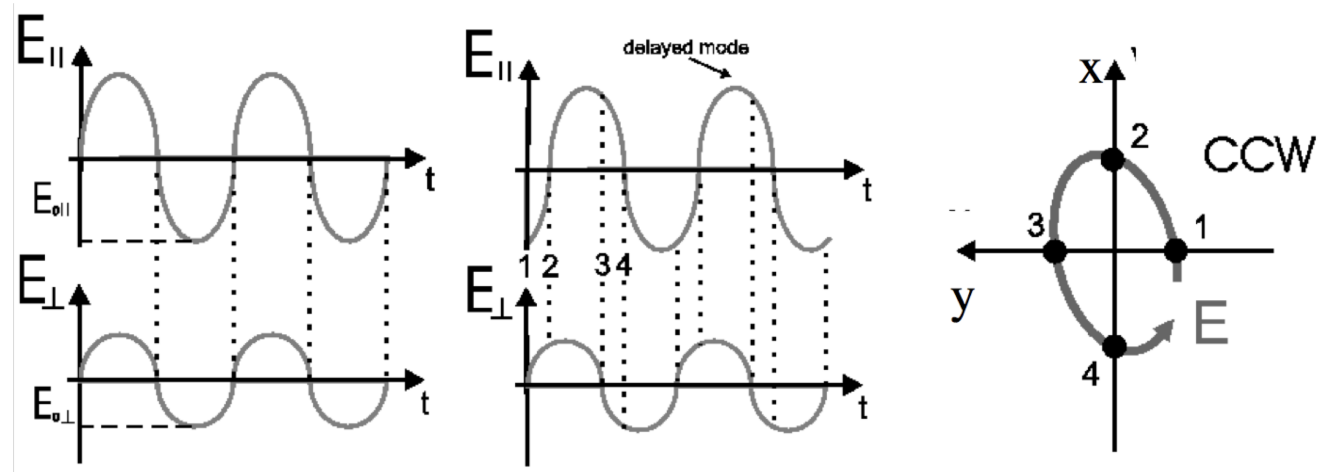

Figure 8: Schematic illustration of how circular polarization is generated by the introduction of a delay between two orthogonal electric field components of an elecromagnetic wave. The panels show the relationship between the two components before and after the delay and the generation of circular polarization.

up to several tenths of a percent, are high enough to make this mechanism implausible. A more likely mechanism is the Faraday conversion of linear-to-circular polarization during propogation through a magnetised plasma; although this mechanism has been studied fairly extensively theoretically and demonstrated to be plausible, but it is difficult to firmly demonstrate its action observationally.

Similar to Faraday rotation, Faraday conversion can occur when a polarized electromagnetic wave passes through a magnetized plasma. In order for Faraday conversion to operate, the observed linear polarization electric (E) vector must have non-zero components both parallel to $\left(\mathbf{E}_{\|}\right)$and perpendicular to $\left(\mathbf{E}_{\perp}\right)$ the magnetic field in the conversion region projected onto the sky, $\mathbf{B}_{\text {conv }}$. The electric-field component $\mathbf{E}_{\|}$excites oscillations of free charges in the plasma, while $\mathbf{E}_{\perp}$ cannot, since the charges are not free to move perpendicular to $\mathbf{B}_{\text {conv }}$. This leads to a delay between $\mathbf{E}_{\|}$ and $\mathbf{E}_{\perp}$, manifest as the introduction of a small amount of CP; the sign of the $\mathrm{CP}$ depends on the relative phase of $\mathbf{E}_{\|}$and $\mathbf{E}_{\perp}$.

Faraday conversion is a direct analog of Faraday rotation, but unlike Faraday rotation, Faraday conversion does not depend on the sign of the free charges involved. If the polarization $\mathbf{E}$ vector is entirely parallel to the ambient magnetic field in the conversion region, it will be entirely absorbed and re-emitted, so that no delay, and hence no circular polarization, will be generated. Similarly, if $\mathbf{E}$ is entirely orthogonal to the ambient magnetic field, it cannot be absorbed at all, so that again, no circular polarization will be generated.

\section{Conclusion}

This paper has not pretended to be a rigorous treatment of the various physical phenomena considered here, but may be helpful to students beginning studies of astrophysical objects in which they are manifest, such as AGN and microquasars. In addition, Faraday rotation can provide a powerful tool for studies of the magnetized plasma giving rise to this effect, and as such can play an important role in studies of cosmic magnetism.

Further and more complete information about synchrotron radiation and other non-thermal radiation mechanisms, as well Faraday rotation, can be found in the textbook by Rybicki and 
Lightman (1986). Burn (1966) has written a classic monograph on Faraday depolarization. A thorough mathematical description of Faraday conversion is given by Jones \& O'Dell (1977) and Jones (1988).

\section{References}

[1] Burn B. J. 1966, MNRAS, 133, 67

[2] Jones T.W. 1988, ApJ, 332, 678

[3] Jones T.W. \& O’Dell S.L. 1977, ApJ, 214, 522

[4] Rybicki G. B. \& Lightman, A. P. 1986, Radiative Processes in Astrophysics (Wiley). 